In children, the prevalence of allergic diseases was higher than expected for those with type $1 \mathrm{DM}$, with $44.4 \%$ in one study having atopic eczema (common sensitivities included Dermatophagoides pteronyssinus, D. farinae or Blomia tropicalis). In a survey of veterinary dermatologists, West Highland White Terriers were repeatedly mentioned as the breed most likely to have concurrent AD and DM. This is a breed that also has a high incidence of secondary Malassezia dermatitis and bacterial pyoderma.

Control of secondary infections is important to achieving good control of DM. Stress associated with pruritus impedes control of hyperglycaemia, therefore anti-pruritic therapy is important. Topical therapies to improve skin barrier function, control infections and alleviate pruritus should be prescribed. Omega-3 fatty acids, antihistamines and tricylic antidepressants are beneficial in many patients. CsA is often prescribed as an alternative to corticosteroids; however, CSA has been shown to enhance gluconeogenesis, decrease serum insulin concentrations and reduce glucose transporter 4 on adipocytes, and therefore may have diabetogenic effects in some patients. Corticosteroids are diabetogenic in both dogs and cats, therefore judicious use is needed when required in the management of a patient with DM. Oclacitinib is a new j-stat inhibitor that shows promise for controlling pruritus in dogs with $\mathrm{AD}$ and DM. Other new therapies may be on the horizon; for example, Morus alba is reported to be antidiabetic and has inhibitory effects on AD-like lesions in a murine model. Further testing is certainly needed.

\section{KEY LEARNING POINTS}

- Diabetes mellitus and atopic dermatitis are complex diseases that involve interactions of genetic and environmental factors and inflammation
Stress from cutaneous inflammation, infections and pruritus impede control of hyperglycaemia in patients with concurrent diabetes mellitus and atopic dermatitis - Topical therapy is important in improving skin barrier function, controlling infections and alleviating pruritus in dogs with atopic dermatitis

- Allergen-specific immunotherapy should be recommended for patients with concurrent diabetes mellitus and atopic dermatitis

\section{MCQS}

1. Which topical product alleviates pruritus by stabilising neuronal membranes of peripheral nerve endings?
a. Betamethasone
b. Pramoxine
c. Tacrolimus
d. Amitriptyline

2. Which is the preferred treatment for long-term management of atopic dermatitis in a diabetic cat or dog?
a. Ciclosporin A
b. Triamcinolone
c. Trimeprazine-prednisolone
d. Allergen-specific immunotherapy

3. Which medication is a Janus kinase inhibitor that inhibits the function of cytokines, such as IL-31, that are pro-inflammatory and have a role in allergic responses and pruritus?
a. Ciclosporin
b. Oclacitinib
c. Tacrolimus
d. Capsicum

\title{
Open fractures and skin wounds
}

\section{Brian Beale}

The initial treatment of an open fracture is likely to affect the final outcome. The fundamental goals to consider every time we see an open fracture are to:

\section{Prevent sepsis \\ Promote union \\ Restore function}

\section{CLASSIFICATION OF OPEN FRACTURES}

- Type I: these are injuries associated with a fracture where the wound is created from the inside to the outside. A sharp bone fragment penetrates the skin creating a puncture wound. The damage to the soft tissues is minimal and there is no soft tissue crushing injury. The fracture configuration is usually short

oblique, transverse or spiral with little or no comminution

- Type II: with these injuries, the insulting agent generating the fracture is from an exterior source, creating soft tissue damage at the site of the impacted area. There is mild to moderate tissue damage, but relatively little avascular or devitalised tissue Type III: these injuries are characterised by extensive soft tissue damage with devitalisation and major contamination. The soft tissue damage is full thickness, involving skin, muscles and neurovascular structures. These fractures are often highly comminuted and are generally caused by a highenergy trauma

\section{PATIENT ASSESSIMENT}

Up to $40 \%$ of patients with orthopaedic injuries have concurrent thoracic or abdominal injuries. The initial assessment consists of rapid evaluation of the respiratory and circulatory function. Treatment of shock and/or respiratory distress is initiated immediately. Open wounds are covered with sterile dressing and distal factures should be splinted to prevent further soft tissue injury and discomfort for the patient. 


\section{Congress 2015}

\section{WOUND MANAGEMENT}

Wound management includes surgical debridement with copious lavage, followed by application of a sterile bandage. During debridement of a wound, fascia and fat are expendable, muscles and skin should be managed in a conservative manner, and ligaments, tendons and bone should be left unless removal is absolutely necessary.

\section{FRACTURE REPAIR}

Fracture fixation is undertaken immediately after an aggressive debridement has converted the contaminated or infected wound in a clean-contaminated wound. Open fractures can be repaired with bone plates, interlocking nails or external fixators (ESFs). Intramedullary pins and cerclage wiring should be avoided. Internal fixation requires some consideration; it is not uncommon to hear that internal fixation should be avoided to prevent the introduction of a foreign body that will increase the likelihood of infection. As numerous authors have shown, this not true and it is safe to perform internal fixation on open fractures after appropriate debridement. External fixation offers undoubted advantages for the management of many open fractures and is probably the method of choice for fractures distal to the elbow and stifle involving extensive soft tissue damage.

\section{KEY LEARNING POINTS}

- Open fractures should be treated as soon as possible to decrease the risk of infection

- The severity of open fractures is graded I-III, from least to greatest severity
Bone plates and external fixators are useful for repair of open fractures

\section{MCQS}

1. Which of the following is TRUE about open fractures?

a. Cannot be repaired with minimally invasive surgery due to the inability to attach the locking jig

b. Cannot be attached to the bone with cortical screws

c. Are graded type I (most severe) to type III (less severe)

d. Should be treated as soon as possible to lessen the chance of infection

2. Which of the following is TRUE about patients with open fractures?

a. Should have fracture repair as soon as possible despite the presence of concurrent thoracic or abdominal injuries

b. Should have open wounds covered with a sterile dressing after appropriate debridement of devitalised tissue

c. Should not be treated with a temporary splint due to the high likelihood of additional vascular compromise

d. Have a poor prognosis and amputation is recommended

3. Which implant is least indicated to repair an open fracture?

a. Intramedullary pin and cerclage wires

b. Bone plate and screws

c. Interlocking nails and bolts

d. External fixator

\section{Anaesthetising the patient with a metabolic disease}

\section{Matthew McMillan}

There are several endocrine diseases that are commonly encountered in general practice and occasionally patients with these diseases will require anaesthesia. Each disease offers a number of anaesthetic challenges, having a set of special requirements that should be considered before performing anaesthesia. The common approach to each of these diseases is to ensure any animal with metabolic disease is stabilised as much as the situation allows prior to anaesthesia. The next step is to consider how the disease could affect anaesthesia (e.g. exacerbate the adverse effects of anaesthetic drugs) and how anaesthesia could affect the disease (e.g. disrupt the stability of the patient) and what can be done about it.

In patients with diabetes mellitus for example, fasting may interfere with the patient's normal feeding and insulin regime. This can lead to hypo- or hyperglycaemia during the perianaesthetic period but may also have knock-on effects with glucose control later on, especially if the patient becomes inappetent. Drugs such as alpha-2 agonists interfere with insulin secretion from the remaining functional pancreas and will complicate blood glucose management, so should be avoided. Hypotension is a recognised additional risk for diabetic patients so doses of acepromazine should be reduced and doses of induction and maintenance agents should be minimised. Careful timing of anaesthesia, monitoring of blood glucose, adjustment of insulin doses and judicious glucose supplementation can all be utilised alongside optimal analgesia to improve recovery and return to normal feeding and insulin patterns.

Dysfunction of the thyroid gland poses a different set of challenges. Even well managed hypothyroid patients have a tendency towards a hypodynamic cardiovascular system. This minimises their ability to compensate following challenges to the cardiovascular system, such as vasodilation and hypovolaemia. Bradycardia and hypotension are commonly encountered and a reduction in metabolic capabilities can also cause hypothermia and prolonged recoveries (through prolonged drug action). In addition, muscle weakness can lead to hypoventilation and reduced respiratory capacities. Hyperthyroid patients on the other hand are unpredictable and are at considerable risk of tachyarrhythmia, hypertension and hypothermia. Concurrent kidney insufficiency may also be problematic, not to mention the fact that hyperthyroid cats are often very difficult to restrain and handle. 